\title{
Designing PID controller for position control with disturbance
}

\begin{abstract}
In this study, a position control scheme of a radio telescope (antenna) is presented. The controller is designed in MATLAB Simulink environment where the antenna model is created. PID controller is proposed to satisfy the requirement of high accuracy position control. However, with the presence of wind disturbance in the system model, it will disrupt the operation of the antenna since it is one of major factor that affects the pointing accuracy. Thus, with assistance of PID controller, it is expected to reduce the effect of this disturbance to the system.
\end{abstract}

Keyword: PID controller; Position control; Radio telescope 